Bulletin of the Natural History Museum, 2018, 11: 215-226.

Received 09 Nov 2018; Accepted 12 Dec 2018.

doi:10.5937/bnhmb1811215P

UDC: 597.754(497.11)

Original scientific paper

\title{
NEW HETEROPTERA IN SERBIA
}

\author{
LJILJANA PROTIĆ
}

Natural History Museum, 11000 Belgrade, Serbia, e-mail: ljilja.protic@gmail.com

This paper presents 13 species new for Heteroptera fauna of Serbia: Family Miridae: Apolygus rhamnicola (Reuter, 1885), Dichrooscytus intermedius Reuter, 1885; Lygocoris viridis (Fallén, 1807); Euryopicoris nitidus (Meyer-Dür, 1843); Halticus major Wagner 1951; Strongylocoris atrocoeruleus (Fieber, 1864); Megalocoleus naso (Reuter,1879); Orthonotus pseudoponticus Josifov, 1964; Orthonotus syriacus (Puton, 1881); Parapsallus vitellinus (Scholtz, 1847); Psallus (Psallus) varians varians (Herrich-Schaeffer, 1841); family Lygaeidae: Lygaeus simulans Deckert, 1985; family Pentatomidae: Aelia melanota Fieber, 1868. Also presented are the first data on Lygaeus simulans for Montenegro.

Key words: Heteroptera, Serbia, Balkan Peninsula

\section{INTRODUCTION}

Continuous studies of Heteroptera in Serbia are based on identification and revision of specimens from the Study Collection of the Natural History Museum in Belgrade and processing of fresh material collected in the last several years. This paper presents 13 new species for Heteroptera fauna of 
Serbia. New data are also presented for species Lygaeus simulans Deckert in Macedonia and the first record for Heteroptera fauna of Montenegro.

\section{MATERIAL AND METHODS}

The studied specimens were collected in various localities throughout Serbia (35 the whole), with one additional specimen for each Macedonia and Montenegro. Most specimens were collected by the method of mowing with a sweep net, while some were shaken off tree branches or individually sampled. All specimens are stored at the Natural History Museum in Belgrade within the Study Collection of Heteroptera.

\section{RESULTS}

MIRIDAE Hahn, 1833

MIRINAE Hahn, 1833

Mirini Hahn, 1833

\section{Apolygus rhamnicola (Reuter, 1885)}

New record - Serbia: Besna Kobila 27.07.2018. 1f legs. A. Stojanović $\&$ M. Jovanović. Besna Kobila belongs to Rhodopes mountain system. It is situated on the route from Vranje and Kriva Feja toward Bosilegrad. This specimen was collected in sweep net in the meadows above Kriva Feja (at about $1300 \mathrm{~m}$ above sea level).

European species. This is the first record for Balkan Peninsula.

Species of the same genus recorded in Serbia and other Balkan countries include Apolygus limbatus (Fallén), Apolygus lucorum (Meyer-Dür) and Apolygus spinolai (Meyer-Dür). They mostly inhabit mesophilous meadows.

\section{Dichrooscytus intermedius Reuter, 1885}

New record - Serbia: Mt. Zlatar, Hotel "Panorama" $1230 \mathrm{~m}$ a.s.l. If 22.07.1987. leg. Lj. Protić on Picea excelsa (Lam.).

Mt. Zlatar belongs to Dinaric mountain chain that reaches the Adriatic Sea. Spruce is dominant in the tree stratum of forests. Single trees of beech appear only in a few stands, while fir was recorded only in one tree stand (Novaković \& Cvjetičanin 2014).

Balkan Peninsula: Bulgaria (Josifov 1974); Albania (Josifov 1986); Slovenia (Gogala \& Gogala 1986, 1989); Romania (Togânel 2006); Montenegro (Protić 2016).

Euro-Siberian boreo-montane species. Lives on conifer trees: Picea sp., Abies sp., Larix sp. 


\section{Lygocoris viridis (Fallén, 1807)}

Lygus (Neolygus) viridis Fallén, 1807

New record - Serbia: Mt. Rudnik under Mali Šturac 970 m a.s.l. If 11.08.2018. leg. A. Stojanović on Acer pseudoplatanus L.

The locality is situated close to the top, at the slope facing away from sun. It is characterized by an old mixed deciduous forest (beech, linden, mountain maple, cherry, hornbeam), while the other side of the mountain top (exposed to direct sunshine) is under degraded oak forest.

Balkan Peninsula: Croatia (Novak \& Wagner 1951); Macedonia (Göllner-Scheiding 1978); Bulgaria (Josifov 1986); Slovenia (Gogala \& Gogala 1986, 1989).

European species, lives in deciduous trees (Alnus sp., Tilia sp.).

ORTHOTYLINAE Van Duzee, 1916 (1865)

Halticini A. Costa, 1853

\section{Euryopicoris nitidus (Meyer-Dür, 1843)}

New record - Serbia: Planina Stolovi, 5m 3f 05.06.2018. legs. A. Stojanović \& M. Jovanović.

Specimens were collected at locality Ravni Sto (1100-1150 m above sea level) at the foothills of the highest peak (Usovica $1272 \mathrm{~m}$ a.s.l.). The habitat includes high-mountain meadows with almost constant wind above the mountain ridge. Sparse woodland of beech and conifers (mostly black pine) descends down the slopes, but the ridge includes plenty of Betula sp., Sorbus sp. and Juniperus communis L. strewn around the meadows which are gradually becoming overgrown with trees. This species was collected with sweep nets, in the same samples with Dimorphocoris sauli Wagner and Euryopicoris nitidus (Meyer-Dür).

Balkan Peninsula: Macedonia (Kormilev 1936); Greece (Günther 1990); Montenegro (Protić et al. 1990).

Palearctic species. Lives on grasses of high-mountain meadows.

\section{Halticus major Wagner 1951}

New record - Serbia: Negotin: Mihajlovac, Djalu Mare 1m 19.07.2017. legs. A. Stojanović \& M. Jovanović

Locality Djalu Mare (Fig. 1) is about 250-300 m above sea level (in the sweep-net zone) and about $5 \mathrm{~km}$ from the bank of Danube. Thermophilic meadow with a few strewn bushes and abandoned vineyards. The whole area includes abandoned arable land: meadows, cereal fields, vineyards, abandoned construction sites, oak woodland, all in process of being reconquered and overgrown by autochthonous vegetation. 
Balkan Peninsula: Croatia (Novak \& Wagner 1955, Balarin 1975); Macedonia (Göllner-Scheiding 1978); Greece (Günther 1990).

European species. Prefers warm habitats. Lives on weeds.

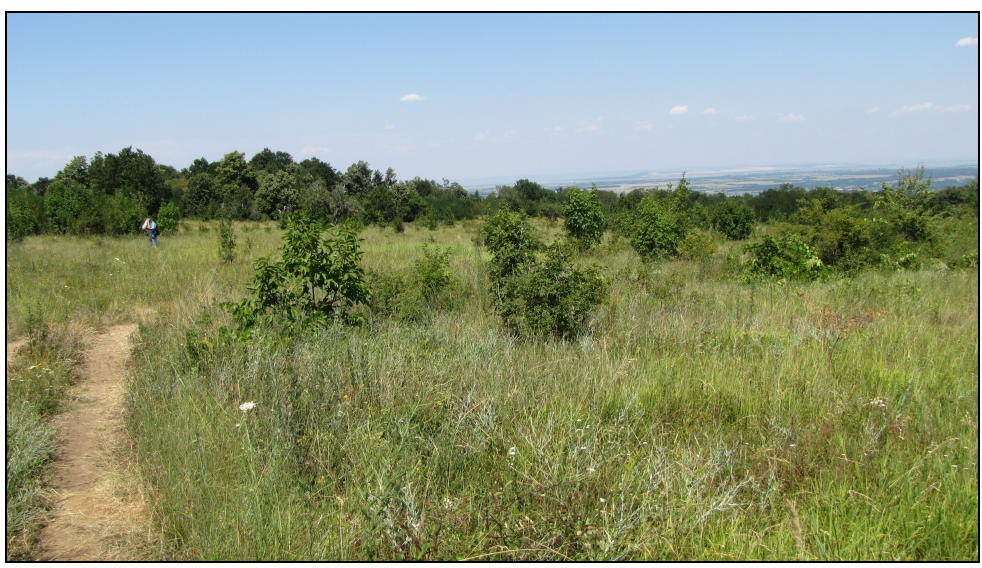

Fig. 1. - Negotin: Mihajlovac, Djalu Mare (Photo: M. Jovanović).

\section{Strongylocoris atrocoeruleus (Fieber, 1864)}

New record - Serbia: Mt. Čemerno: Smrdljuč (Fig. 2) at 1420-1470 m a.s.1. 02.08.2017. 1f legs. A. Stojanović \& M. Jovanović.

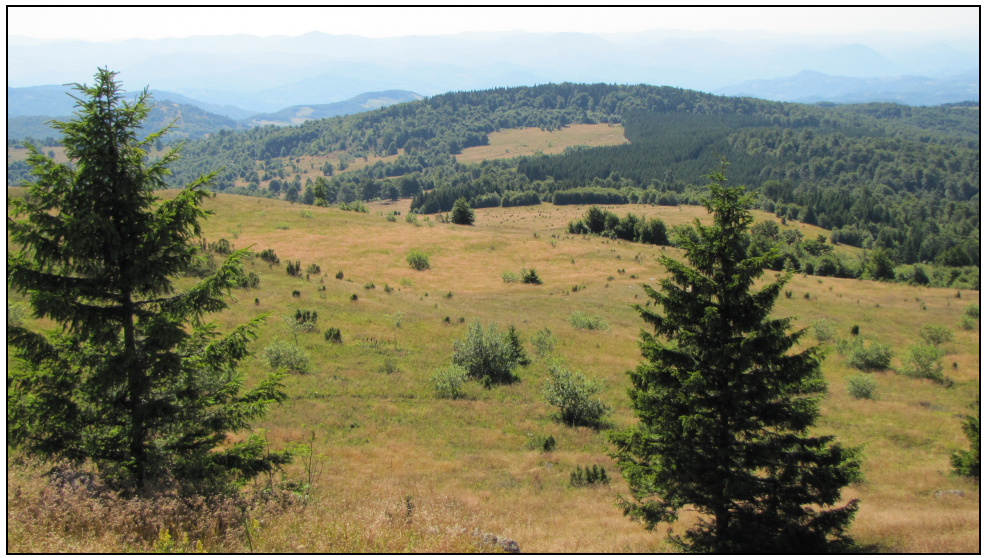

Fig. 2. - Čemerno Mt.: Smrdljuč (Photo: M. Jovanović).

Locality Smrdljuč is a high-mountain meadow at the edge of spruce forest, with a lot of bilberries, while individual oak or beech trees may exist on the slopes.

Balkan Peninsula: Slovenia (Gogala \& Gogala 1986).

European species, also present in North Africa in Morocco (Kerzhner \& Josifov 1999) 
Mountain species, collected on Peucedanum officinale L.

PHYLINAE Douglas \& Scott, 1865

Phylini Douglas \& Scott, 1865

Megalocoleus naso (Reuter,1879)

Amblytylus macedonicus Wagner, 1956

New record - Serbia: Deliblato Sands: Devojački Bunar 140-150 m a.s.1. 19.06.2004. 1f leg. A. Stojanović

Species characteristic for this locality include Festuca sp., Stipa sp. and Koeleria sp. - steppe association - Festucetum vaginatae pogonetum pannonicum typicum (Stjepanović-Veseličić, 1953).

Balkan Peninsula: Macedonia (Wagner 1956, 1962; Matocq \& PluotSigwalt 2012); Bulgaria (Josifov 1986); Greece (Matocq \& Pluot-Sigwalt 2012).

According to Kerzhner \& Josifov (1999) and Matocq (2004) outside of Balkan Peninsula it was recorded in France (Corsica), Hungary, Italy (Sardinia), Slovakia, Turkey, Ukraine, and Iraq.

Linnavuori (1993) stated that it is distributed in Pontian-Mediterranean region, although in the last several years its range is expanding northward. It was recorded in Austria (Rabitsch 2001; Rabitsch \& Matocq 2005), and later also in Germany (Damken \& Stöckmann 2016).

Kerzhner \& Josifov (1999) also mentioned records from Croatia, although Pajač et al. (2010) did not include that information.

\section{Orthonotus pseudoponticus Josifov, 1964}

New record - Serbia: Pl. Stolovi, high-mountain meadow. 05.06.2018. 10m 10f legs. A. Stojanović \& M. Jovanović.

The specimens were collected on the mountain ridge between Orlovac (944 $\mathrm{m}$ a.s.1.) and the highest peak Usovica (1272 $\mathrm{m}$ a.s.1.), at the locality Ravni Sto (1100-1150 m a.s.1.). They were collected at the same place, together in a single swipe of sweep net, with Euryopicoris nitidus and Dimorphocoris sauli.

Endemic of Balkan Peninsula (Josifov \& Simov 2006).

Bulgaria: Rhodopes, Komuniga, Pirin, Popina Laka, Ali-Botush, Studena (Josifiv 1964); Macedonia: Mt. Karadžica (Göllner-Scheiding 1978); Grecee (Josifov \& Simov 2006).

Mountain species. Josifov (1964) collected this species in the belt between deciduous and coniferous forests. It was collected from late May to late July. 


\section{Orthonotus syriacus (Puton, 1881)}

New record - Serbia: Besna Kobila 27.07.2018. 1f legs. A. Stojanović $\&$ M. Jovanović on Verbascum longifolium Ten. (Fig. 3) along the road, from $1456 \mathrm{~m}$ above sea level to the peak which is at $1909 \mathrm{~m}$ above sea level.

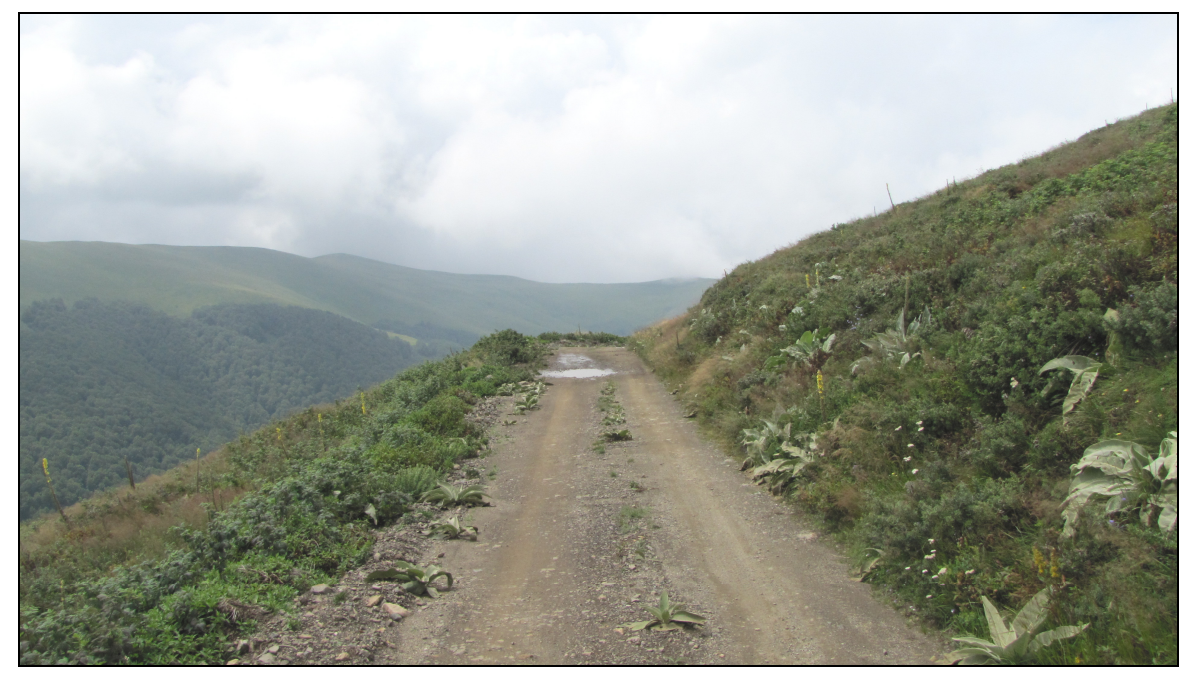

Fig. 3. - Besna Kobila Mt. (Verbascum longifolium) (Photo: M. Jovanović)

The specimen was collected at the abaxial side of leaf, in a young rosette, together with species Adelphophylus balcanicus Kormilev. Population of A. balcanicus was abundant, with more than 10 individuals in each leaf rosette of mullein, which is a cluster of young mulleins that will flower next year.

Kerzhner \& Josifov (1999) listed only Israel (Haifa) for distribution.

In sunny, dry habitats, meadows and stony hills.

\section{Parapsallus vitellinus (Scholtz, 1847)}

Capsus vitelinus Scholtz, 1847

Plagiognathus (Parapsallus) vitellinus (Scholtz, 1846)

New records - Serbia: Tara Mt.: Račanska Šljivovica 29.07.1981. 1f, Tara Mt.: Kaluđerske Bare 02.08.1981. 1f, Tara Mt.: Jelisavčići 04.08. 1981. 1f; Tara Mt.: Kozje Stene 28.07.1988. 1f 1 larva, Tara Mt.: Zaovine, Malo Jezero 03.08.1988. larva, Tara Mt.: Mitrovac 24.07.1989. 1m 1f leg. Lj. Protić. At Tara it was collected on Picea excelsa (Lam.), at the altitude of 1100-1250 $\mathrm{m}$ above sea level.

The prevalent forest association at Tara is mixed forest association Piceo-Fago-Abietetum Čolić 1965 (Cvjetićanin \& Novaković 2010). 
Balkan Peninsula: Bulgaria (Josifov 1986); Macedonia (Josifov 1986); Slovenia (Gogala \& Gogala 1986); Romania (Kerzhner \& Josifov 1999).

Euro-Siberian species (Duwal et al. 2013), living on conifers.

\section{Psallus (Psallus) varians varians (Herrich-Schaeffer, 1841)}

New record - Serbia: Dukat Mt. Crnook - Mečit (Fig. 4) $2 \mathrm{~m}$ 02.07.2018. legs. A. Stojanović \& M. Jovanović.

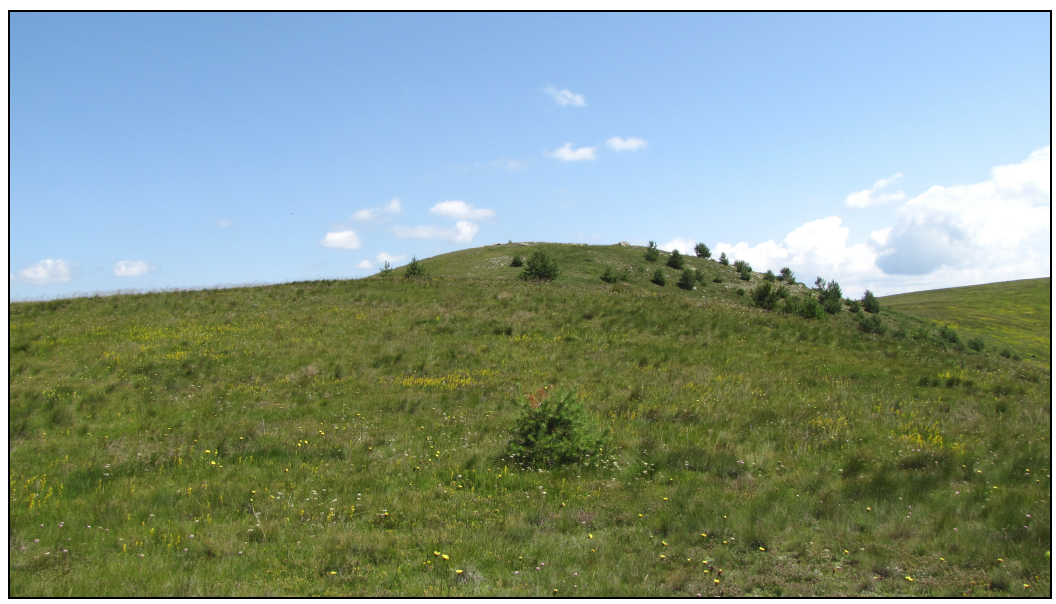

Fig. 4. - Dukat Mt. Crnook - Mečit Pinus nigra (Photo: M. Jovanović)

Samples were collected on the slope at the highest peak of Mt. Dukat Crnook (1881 m above sea level), from individual scattered trees of Pinus nigra J. F. Arnold at altitudes of 1570-1600 m above sea level.

Balkan Peninsula: Albania (Josifov 1986); Bulgaria, Macedonia (Josifov 1986, 1999); Montenegro (Protić et al. 1990); Croatia (Protić 1998); Greece (Kerzhner \& Josifov 1999);

High mountain species, on Fagus silvatica L., Fraxinus excelsior L. Quercus sp., Pinus sp., Picea sp.

The range includes Europe: widespread (Wagner 1962a, 1975; Aukema et al. 2013), in Asia: Azerbaijan, Iran, Turkey (Asian part), Georgia (Linnavuori 2007, Aukema et al. 2013), and in Africa: Tunisia (Linnavuori 2007).

LYGAEIDAE Schilling, 1829

LYGAEINAE Schilling, 1829

\section{Lygaeus simulans, Deckert 1985}

New records - Serbia: Stara Planina: Topli Do 13.07.1979. 1m leg. M. Brajković; Grocka 18.07.1982. 1m leg. Lj. Anđus; Mt. Rudnik 08.1983. 1m 
leg. A. Sigunov; Djerdap: Veliki Štrbac 27.06.1985. 1f leg. Lj. Protić; Ramsko-Golubačka Peščara: Vince 29.06.1985. 1m leg. Lj. Protić; Deliblato Sands: Dubovac 14.07.1987. 1m; Deliblato Sands: Tilva 16.06.1988. $2 \mathrm{~m}$ leg. Lj. Protić; Popović 08.08.1998. 1f leg. A. Stojanović; Surduk 27.06. 1998. 2m 3f; 21.08.1999 1m; 11.06.2016. 1f leg. A. Stojanović; Novi Beograd: confluence of Galovica 03.07.1999. 1m 2f leg. A. Stojanović; Deliblato Sands: Devojački Bunar 09.08.2003; 2f; 19.06.2004. 2f; 19.08. 2006. 3f; 12.05.2007. 1f; 12.07.2008. 1f leg. A. Stojanović; Grocka: Bega1jica 24.04.2004. 1m leg. A. Stojanović; Mala Moštanica: Žuto Brdo 07.08. 2004. $1 \mathrm{~m}$ leg. A. Stojanović; Beljanica; Beljanička Kapa on Cyanidium vincetoxicum 21.07.2004. 2m 2f leg. M. Vujanić; Beograd: Veliko Selo 28.06.2008. 1f leg. A. Stojanović; Stari Slankamen: Koševac 10.05.2008. 1m; 11.04.2009. 1f leg. A. Stojanović; Podlužje: Boljevci, Crni lug 21.06.2008. 2f leg. A. Stojanović; Mt. Rudnik 09.07.2011. 1m leg. A. Stojanović; Mt. Rudnik: Cvijićev Vrh 20.07.2013. 1f leg. A. Stojanović; Mt. Stolovi 02.09.2015. 1f legs. A. Stojanović \& M. Jovanović; Studena Mt. 04.09.2015. 2m legs. A. Stojanović \& M. Jovanović.

Note. The entomological box labelled Lygaeus equestris also includes specimens of $L$. simulans which were identified as $L$. equestris.

This paper includes a list of 20 localities where L. simulans was collected, from steppe habitats of Deliblato Sands, to lowland meadows along river Danube, to mountain pastures. This species has a wide ecological spectrum - it is a eurobiont.

In addition to specimens from Serbia, the revised material also includes one specimen from Macedonia - Mt. Galičica (1600 m above sea level), one male, collected by former curator of Natural History Museum Miloje Brajković on August $11^{\text {th }} 1977$.

The second specimen collected outside the territory of Serbia is a female from Montenegro, collected at Mt. Durmitor, locality Mlinski Potok, at July $17^{\text {th }} 1987$. leg. M. Živković. This was the first record for fauna of Montenegro.

Palearctic species.

PENTATOMIDAE Leach, 1815

PenTATOMINAE Leach, 1815

Aelini Douglas \& Scott, 1865

Aelia notata Rey, 1887

New record - Serbia: Dukat Mt. Crnook - Mečit, 03.07.2018. 5m 5f legs. A. Stojanović \& M. Jovanović. 
Mt. Dukat is formed out of crystalline schist and granite. The mountain ridge is covered in meadows. The specimens were collected at locality Mečit at the altitude of $1630 \mathrm{~m}$ above sea level. Due to intensive rain several days before collecting, method of sweep net produced poor results, but the collector was under impression that $A$. notata is not a rare species in this locality, and instead it is common and present for a longer period of time.

Balkan Peninsula: Serbia.

Distribution in Europe: France, Spain, Ukraine (Derzhansky \& Péricart 2005; Derzhansky et al. 2006).

\section{Acknowledgement}

I would like to thank Aleksandar Stojanović and Miroslav Jovanović (Natural History Museum Belgrade) for collecting the specimens. I am particularly grateful to colleague Stojanović for useful suggestions and providing data on locations where he collected the specimens.

\section{REFERENCES}

Aukema, B., Rieger, Ch., Rabitsch, W. (2013): Catalogue of the Heteroptera of the Palaearctic Region. VI. Supplement. - The Netherlands Entomological Society, Amsterdam.

Balarin, I. (1975): Inventarizacija biljnih bolesti i štetnika na graničnim područjima. - Republički sekretarijat za poljoprivredu, prehrambenu industriju i šumarstvo SR Hrvatske, Zagreb 3(1-4): 40-67.

Cvjetićanin, R., Novaković, M. (2010): Floristički diverzitet šume jele, bukve i smrče (Piceo-Fago-Abietetum Čolić 1965) u nacionalnom parku „Tara“. Glasnik Šumarskog fakulteta 102: 129-144.

Damken, C., Stöckmann, L. (2016): Erstnachweis der Weichwanze Megalocoleus naso (Reuter, 1879) für Deutschland. - Heteropteron 46: 5-6.

Deckert, J. (1985): Über Lygaeus simulans spec. nov. und L. equestris (L.), zwei nahe verwandte palärktische Lygaeinae (Heteroptera, Lygaeidae). - Mitteilungen aus dem Zoologischen Museum in Berlin 61: 273-278. [http://dx.doi.org/ 10.1002/mmnz.19850610203]

Derzhansky, V., Péricart, J., (2005): Hémiptères Pentatomoidea Euro-méditerrnéens vol. I Généralités Systématique: Première Partie, Faune de France (France et régions limitrophes). - Fédération des Sociétés des Sciences Naturelles, Paris.

Derzhansky, V., Péricart, J. \& Pluot-Sigwalt, D. (2006): Notes on synonymy and distribution of some species of the genus Aelia (Heteroptera: Pentatomidae). Zoosystematica Rossica 15(2): 298. 
Duwal, R. K., Jung, S., Lee, S. (2013): Resurrection of the genus Parapsallus Wagner (Hemiptera: Heteroptera: Miridae: Phylinae: Phylini). - Zootaxa 3647(4): 597-600.

Gogala, A., Gogala, M. (1986): Seznam vrst stenic ugotovljenih v Sloveniji (Insecta: Heteroptera). - Biološki Vestnik 34: 21-52.

Gogala, A., Gogala, M. (1989): True Bugs of Slovenia (Insecta: Heteroptera). Biološki Vestnik 37: 11-44.

Göllner-Sheiding, U. (1978): Beitrag zur kenntnis der Heteropterenfauna Mazedoniens. - Acta Musei Macedonici scientiarum naturalium Skopje 15, 6(131): 145-150.

Günther, H. (1990): Contribution to the Heteroptera Fauna of the Balkan peninsula. - Scopolia, Suppl. 1: 39-52.

Josifov, M. 1964 Über die Gattung Orthonotus Stephens, 1829. - Reichenbachia 4 (18): 151-156.

Josifov, M. (1974): Beitrag zur Systematic der paläärktischen Dichrooscytus-Arten (Heteroptera, Miridae). - Reichenbachia 5 (20): 149-173.

Josifov, M. (1986): Verzeichnis der von Balkanhalbinsel bekannten Heteropterenarten (Insecta, Heteroptera). - Faunistische Abhandlungen Staatliches Museum für Tierkunde Dresden 14 (6): 61-93.

Josifov, M. (1999): Heteropterous insects in the Sandanski-Petrich Kettle, Southwestern Bulgaria - Historia naturalis bulgarica 10: 35-66

Josifov, M., Simov, N. (2006): Endemism among the Heteroptera on the Balkan Peninsula. In: Rabitsch, W. (ed.): Hug the bug - For love of true bugs. Festschrift zum 70. Geburtstag von Ernst Heiss. - Denisia 19: 879-898.

Kerzhner, I. M., Josifov, M. (1999): Cimicomorpha II. Miridae. In: Aukema, B. \& Rieger, Ch. (eds): Catalogue of the Heteroptera of the Palaearctic Region 3. Netherlands Entomological Society, Amsterdam.

Kormilev, N. (1936): 1. prilog poznavanju Hemiptera - Heteroptera Jugoslavije (Južna Srbija i Srbija). [First contribution to knowledge of Hemiptera Heteroptera of Yugoslavia (Southern Serbia and Serbia)]. - Glasnik skopskog naučnog društva 17(5): 29-54.

Linnavuori, R. E. (1993): Hemiptera of Iraq. III. Heteroptera, Miridae (Phylinae). Entomologica Fennica 4: 253-271.

Linnavuori, R. E. (2007): Studies on the Miridae (Heteroptera) of Gilan and the adjacent provinces in northern Iran. II. List of species. - Acta Entomologica Musei nationalis pragae 47: 17-56.

Matocq, A. (2004): Revue des espèces attribuées au genre Megalocoleus REUTER, 1890 (Heteroptera: Miridae). - Annales de la Société Entomologique de France (N.S.) 40(1): 69-101.

Matocq, A., Pluot-Sigwalt, D. (2012): Revision des Amblytylus et essai de mise au point sur les genres Amblytylus Fieber et Megalocoleus Reuter (Heteroptera: Miridae: Phylinae). - Annales de la Société Entomologique de France (N.S.) 48: $123-154$.

Novak, P., Wagner, E. (1951): Prilog poznavanju faune Hemiptera Dalmacije (Hemiptera - Heteroptera). - Godišnjak Biološkog instituta u Sarajevu 4 (1): 59-80. 
Novak, P., Wagner, E. (1955): II Prilog poznavanju faune Hemiptera Dalmacije (Hemiptera- Heteroptera). - Zaštita bilja 30, Dodatak Prilozi entomofauni Jugoslavije: $1-10$.

Novaković, M., Cvjetičanin, R. (2014): Fitocenološke karakteristike čistih i mešovitih šuma smrče na Zlataru. - Šumarstvo 3: 153-162.

Pajač, I., Barić, B., Milošević, B. (2010): Katalog stjenica (Heteroptera: Miridae) Hrvatske. - Entomologica Croatica 14 (1-2): 23-76.

Protić, Lj. (1998): Catalogue of the Heteroptera fauna of Yugoslav countries. Part one. - Natural History Museum, Belgrade. Special Issue 38: 1-215.

Protić, Lj. (2016): Checklist of Heteroptera of Montenegro. - Ecologica Montenegrina, Special Issue 7: 350-393.

Protić, Lj., Gogala, A., Gogala, M. (1990): Heteroptera (Insecta) In: Nonveiller, G. (ed.): Fauna Durmitora 3. - Crnogorska Akademija nauka i umjetnosti, Posebna izdanja 23(14): 279-313.

Rabitsch, W. (2001): Zur Wanzenfauna (Insecta, Heteroptera) im nördlichen Steinfeld. - Stapfia 77: 159-173.

Rabitsch, W., Matocq, A. (2005): Megalocoleus naso (Reuter, 1879), new to Austria. - Beiträge zur Entomofaunistik 6: 166-167.

Stjepanović-Veseličić, L. (1953): Vegetacija Deliblatske peščare. - Srpska akademija nauka i umetnosti, Posebna izdanja 216, Institut za ekologiju i biogeografiju 4: $1-113$.

Togânel, F. (2006): Heteroptere din colecia "Bela Kis" conservate la Muzeul de ştinţele naturii din Tg. Mureş (Heteroptera: Miridae) [True bugs of "Bela Kis" collection preserved in the Natural sciences museum of Tg. Mure (Heteroptera: Miridae)]. Muzeul Olteniei Craiova. Oltenia. Studii şi comunicări. - Ştiinţele Naturii 22: 128-134.

Wagner, E. (1956): Amblytylus macedonicus nov. spec. (Hem. Heteropt. Miridae). - Bollettino della Società Entomologica Italiana 86: 117-120.

Wagner, E. (1962): 2. Beitrag zur Heteropteren - fauna Macedoniens (Hem. Het.). - Fragmenta Balcanica. Musei Macedonici Scientiarum naturalium Skopje 4, 15(99): 115-122.

Wagner, E. (1962a): Über Psallus varians Herrich-Schaeffer 1842 (Hem. Het. Miridae). - Nachrichtenblatt der Bayerischen Entomologen 11: 26-31.

Wagner, E. (1975): Die Miridae Hahn, 1831, des Mittelmeerraumes und der Makaronesischen Inseln (Hemiptera, Heteroptera). - Entomologische Abhandlungen, Supplement 40: 1-484. 


\title{
НОВЕ ХЕТЕРОПТЕРЕ У СРБИJИ
}

\author{
ЉИљАНА ПРОТИЋ
}

\section{Р Е 3 И М Е}

У раду је објављено 13 нових врста за фауну Heteroptera Србије. Фамилија Miridae: Apolygus rhamnicola (Reuter, 1885), Dichrooscytus intermedius Reuter, 1885; Lygocoris viridis (Fallén, 1807); Euryopicoris nitidus (Meyer-Dür, 1843); Halticus major Wagner 1951; Strongylocoris atrocoeruleus (Fieber, 1864); Megalocoleus naso (Reuter,1879); Orthonotus pseudoponticus Josifov, 1964; Orthonotus syriacus (Puton, 1881); Parapsallus vitellinus (Scholtz, 1847); Psallus (Psallus) varians varians (Herrich-Schaeffer, 1841); фамилија Lygaeidae: Lygaeus simulans Deckert, 1985; фамилија Pentatomidae: Aelia melanota Fieber, 1868.

У збирци Heteroptera Природњачког музеја уочено је да се у кутији где се чувају примерци Lygaeus equestris (Linnaeus, 1758) налази још једна врста, веома слична, а то је Lygaeus simulans, Deckert, 1985. У ревидираном материјалу пронађени су и примерци уловљени изван граница Србије, у Црној Гори и Македонији. Примерак са Дурмитора је први податак за фауну Heteroptera Црне Горе. 